\title{
In vitro and in vivo Evaluation of Ibuprofen Nanosuspensions for Enhanced Oral Bioavailability
}

\author{
Mohsen Hedaya Farzana Bandarkar Aly Nada \\ Department of Pharmaceutics, Faculty of Pharmacy, Kuwait University, Kuwait, Kuwait
}

\section{Highlights of the Study}

- Drug solubility and dissolution are essential factors in determining the rate and extent of oral drug absorption.

- Most new drugs have poor aqueous solubility and hence poor bioavailability.

- Ultrahigh-pressure homogenization proved to be a useful technique in improving the solubility and dissolution rate of ibuprofen.

- We observed better pharmacokinetic parameters for IBU nanosuspensions, compared to the reference marketed suspension.

\section{Keywords}

Nanosuspension • Ultrahomogenization · Bioavailability · Ibuprofen · Rabbits

\footnotetext{
Abstract

Introduction: The objectives were to prepare, characterize, and evaluate different ibuprofen (IBU) nanosuspensions. Methods: The nanosuspensions produced by ultrahomogenization were compared with a marketed IBU suspension for particle size, in vitro dissolution, and in vivo absorption. Five groups of rabbits were orally administered with $25 \mathrm{mg} /$ $\mathrm{kg}$ of IBU nanosuspension, nanoparticles, unhomogenized suspension, marketed product, and untreated suspension. A sixth group received $5 \mathrm{mg} / \mathrm{kg}$ IBU intravenously. Blood samples obtained were analyzed by chromatography. Results: The nanosuspensions showed significant decrease in particle size. Polyvinylpyrrolidone (PP) K30 profoundly increased aqueous solubility of IBU. Addition of Tween 80 (TW), in
}

equal amount as PP (IBU:PP:TW, 1:2:2 w/w), resulted in much smaller particle size and better dissolution rate. The $C_{\max }$ values achieved were $14.8 \pm 1.64,11.1 \pm 1.37,9.01 \pm 0.761,7.03$ \pm 1.38 , and $3.23 \pm 1.03 \mu \mathrm{g} / \mathrm{mL}$, and the $t_{\max }$ values were $36 \pm$ $8.2,39 \pm 8.2,100 \pm 17.3,112 \pm 15$, and $105 \pm 17 \mathrm{~min}$ for the nanosuspension, nanoparticle, unhomogenized suspension, marketed IBU suspension, and untreated IBU suspension in water, respectively. Bioavailability of the different formulations relative to the marketed suspension was found to be in the following sequence: nanosuspension > unhomogenized suspension $>$ nanoparticles $>$ untreated IBU suspension. Conclusion: IBU/PP/TW nanosuspension showed enhanced in vitro and in vivo performance as compared to the marketed product. Nanosuspensions prepared by the ultrahigh-pressure homogenization technique can be used as a good formulation strategy to enhance the rate and extent of absorption of poorly soluble drugs.

(C) 2021 The Author(s)

Published by S. Karger AG, Basel
(C) 2021 The Author(s)

Published by S. Karger AG, Basel

This is an Open Access article licensed under the Creative Common Attribution-NonCommercial-4.0 International License (CC BY-NC) (http://www.karger.com/Services/OpenAccessLicense), applicable to the online version of the article only. Usage and distribution for commercial purposes requires written permission. 


\section{Introduction}

It is a well-known fact that considerable number of compounds under development may fail to reach the market because of their poor water solubility. Many reports have shown that a majority of active compounds under development are classified as poorly soluble with most of them under class II drugs in the Biopharmaceutical Classification System (BCS) [1, 2]. Improving the solubility and/or the rate of dissolution of this class of drugs has been an essential factor in enhancing their rate and extent of absorption after oral administration [3]. Common strategies that have been employed to enhance drug solubility include the use of prodrugs which are drug derivatives with better aqueous solubility that can liberate the active drug in vivo after their absorption [4] and the preparation of salts or different crystal structures of the active moiety that have better water solubility [5]. Also, pharmaceutical processing methods such as crushing [6, 7], micronization [8], spray drying [9], solid dispersions [10-12], complexation [13], and solvent coprecipitation [14] have been utilized to reduce particle size and hence improve drug solubility.

Size reduction and wet comminution techniques have also been successfully utilized for the preparation of nanoparticulate drug delivery systems, as they do not use organic solvents or apply harsh environmental conditions; moreover, they can be scaled up easily [15]. However, nanoparticles resulting from these size reduction techniques tend to agglomerate to form larger particles due to the large increase in particle surface free energy [16]. Thus, during the manufacture of nanoparticles, addition of polymeric stabilizers/surfactants is preferred as they act as surface modifiers and regulate the formation of nanoparticles into the desired form [17-19]. However, there is no systematic approach to choose the optimum stabilizer/surfactant, and the selection is usually dependent on trial and error [17].

Several studies have reported the advantages of the high-pressure homogenization (HPH) technique as an approach for size reduction to the nanoscale. The advantages of this technique include efficiency, simplicity, and the possibility for scale-up to large-scale production [20]. The principle of HPH is based on forcing a drug suspension together with the polymeric stabilizer through the nanosized channel of the ultrahomogenizer under very high pressure. The homogenization cycle is usually repeated several times until the nanoparticles with the desired size are obtained. Important parameters for this process which determine the properties of the resulting nanoparticles are the applied pressure and the number of homogenization cycles. This method has been applied to prepare nanosuspensions of several drugs previously, such as diclofenac, paclitaxel, budesonide, and clofazimine [21-24]. Wang and coworkers [25] reviewed the impact of the biochemical and physiological barriers as well as nanoparticle properties such as size, surface properties, and shape of nanoparticle on bioavailability of drugs. A recent report concluded that polyelectrolyte complexation led to polymeric complexes with large particle size and high polydispersity; in contrast, the $\mathrm{HPH}$ technique provided high uniform polymeric complexes on the nanometric scale [26].

The main objective of the current investigation was to ascertain whether the improved in vitro drug dissolution of the nanosuspensions prepared by using the HPH technique will be reflected in enhancing the bioavailabilty of ibuprofen (IBU). The novelty of this work resides on using a combination of hydrophilic polymers with and without surfactant to achieve enhanced dissolution of the drug. Furthermore, IBU was used in this study as a model drug; however, the results may be applied to many other drugs with similar physicochemical properties, such as ketoprofen (KET), nateglinide, and atorvastatin. In the present study, optimized freshly prepared and characterized IBU nanosuspensions were evaluated in vitro to ensure the reproducibility of the previously reported IBU nanosuspensions. The in vivo performance of the optimum nanosuspension formulations along with their corresponding freeze-dried nanoparticles, untreated IBU suspension, and a marketed IBU suspension was investigated in rabbits.

\section{Methods}

Preparation of Nanosuspensions and Optimization of Homogenization

The detailed steps for the formulation and optimization of IBU (Mallinckrodt Pharmaceuticals, St. Louis, MO, USA) nanosuspensions and nanoparticles have been reported previously [27]. In brief, the nanosuspensions were prepared by $\mathrm{HPH}$ using a pressure of 500 bar for 15 cycles, followed by 750 bar for 15 cycles and finally 1,250 bar for 20 cycles. The optimized formulations containing a suitable polymeric stabilizer and surfactant were IBU:PP (Polyvinylpyrrolidone K30; Fluka, Switzerland) (1:2 w/w) and IBU:PP:TW (Tween 80; Merck, Germany) (1:2:2 w/w). Freshly prepared nanoparticles and nanosuspensions underwent in vitro and in vivo evaluation.

\section{Lyophilization}

The nanosuspension formulated using IBU:PP (1:2 w/w) was lyophilized to produce nanoparticles. The nanosuspension pre- 
pared after the ultrahomogenization step was rapidly cooled below $-50^{\circ} \mathrm{C}$, as described before [27]. The resulting dry nanoparticles were stored in airtight containers for further studies. The nanosuspension formulation containing the surfactant TW could not be lyophilized because of the oily nature of TW which would prevent drying of the suspension to form nanoparticles, instead it resulted in a sticky mass.

\section{Particle Size Analysis}

The average particle size of all suspensions was determined by photon correlation spectroscopy, as detailed previously [27].

\section{$I B U$ Content in the Formulations}

Samples from the nanosuspension, freeze-dried nanoparticles, and the marketed product containing an equivalent of 50 $\mathrm{mg}$ of IBU were added to acetonitrile (ACN), sonicated, suitably diluted, and then analyzed for IBU by a validated ultrafast liquid chromatography (UFLC) method [28]. The chromatograph system comprised a Prominence UFLC pump equipped with a photodiode array detector (Shimadzu, Japan). The data were acquired and processed using Shimadzu LC Solutions software. Prefiltered samples $(10 \mu \mathrm{L})$ were injected into a Shim-pack XRODS column (3.0-mm i.d., 30-mm length, and $2.2-\mathrm{mm}$ particle size) maintained at $30^{\circ} \mathrm{C}$. The mobile phase system consisted of ACN (Merck, Germany):water:orthophosphoric acid (Fluka, Switzerland) in the ratio 50:50:0.1 v/v and was run in isocratic mode at a flow rate of $0.25 \mathrm{~mL} / \mathrm{min}$. The run time was $10 \mathrm{~min}$, and the column effluent was monitored at a wavelength of 222 $\mathrm{nm}$.

\section{In vitro Dissolution Studies}

Dissolution study was performed on samples equivalent to 100 mg of IBU of each of the tested formulation either as freeze-dried product or suspension added quantitatively to the dissolution vessels directly. The study was conducted in different dissolution media: $900 \mathrm{~mL}$ water, simulated gastric fluid, simulated intestinal fluid, and phosphate buffer ( $\mathrm{pH} 7.2 \pm 0.1$ ), using USP type 2 apparatus (Erweka DT 80, GmbH, Germany) [21]. The dissolution medium was maintained at $37^{\circ} \mathrm{C} \pm 0.5^{\circ} \mathrm{C}$ and $100 \mathrm{rpm}$, and the run time was $60 \mathrm{~min}$. Samples of the dissolution medium were withdrawn at 15-, 30-, 45-, and 60-min time intervals, filtered, and analyzed by the UFLC method described earlier for content analysis. Fresh dissolution medium at the same temperature was used to replace the samples at each time interval. All studies were performed in triplicate.

\section{In vivo Evaluation of the Developed Formulations}

The in vivo performance of the developed nanosuspension and other formulations was evaluated by conducting a pharmacokinetic study to compare the rate and extent of IBU absorption after single oral administration in rabbits. The in vivo pharmacokinetic study was performed in New Zealand White rabbits weighing 3.5$4.0 \mathrm{~kg}$. Rabbits were provided by the Animal Resource Center affiliated with the Health Science Center of Kuwait University. They were kept individually in clean stainless steel cages, in a temperature- and humidity-controlled room under 12-h light/dark cycles. The rabbits had free access to water and food before the experiment. The protocol for the animal experiments was approved by the Animal Care and Use Committee at the Health Science Center, Kuwait University, and all the procedures used followed the ac- cepted guidelines for the use of experimental animals in research. Intravenous (IV) administration of IBU and collection of blood samples were done through a catheter (Argyle ${ }^{\mathrm{TM}}$ polyurethane umbilical vessel catheter, $0.8 \mathrm{~mm} \times 25 \mathrm{~cm}$; Covidien Inc., Walpole, MA, USA) inserted into the marginal ear vein of the rabbit. The oral formulations were administered to the rabbits using a pediatric feeding tube after opening the rabbit mouth with a rigid rubber tube that has 2 holes facing each other. At the end of each experiment, the rabbits were euthanized using an overdose of IV pentobarbital.

\section{Study Design}

Six groups of rabbits ( $n=5$ each) received the following treatments: group A received IBU as IV hydroalcoholic $(2: 1 \mathrm{v} / \mathrm{v})$ solution; group $\mathrm{B}$ received the untreated IBU suspended in water orally; group $\mathrm{C}$ received the marketed IBU suspension orally (Sapofen ${ }^{\circledR}$ Junior Pediatric Suspension [Lot No. 994544; manufacturing date - September 2017; expiry date - September 2019]; Spimaco, Kingdom of Saudi Arabia ); group D received the unhomogenized IBU suspension orally; group E received the optimized nanoparticles (IBU:PP) orally; and group F received the optimized nanosuspension (IBU:PP:TW) orally. The IV dose was $5 \mathrm{mg} / \mathrm{kg}$, while all the oral formulations were administered at a dose of $25 \mathrm{mg} / \mathrm{kg}$. After each IBU administration, serial blood samples were obtained with the aid of the marginal ear vain catheter over a period of $8 \mathrm{~h}$. Plasma samples obtained from the blood samples by centrifugation were stored at $-80^{\circ} \mathrm{C}$ until analysis.

\section{Pharmacokinetic Analysis}

The pharmacokinetic parameters for IBU were determined using the noncompartmental method for pharmacokinetic data analysis, utilizing the general methods for parameter estimation [29]. The absolute bioavailability for the different oral formulations was calculated from the observed $\mathrm{AUC}_{0-\infty}$ after oral and IV administration after correction for the administered dose, while the bioavailability of the different oral formulations (test) relative to the bioavailability of the marketed suspension (reference) $\left(F_{\mathrm{R}}\right)$ was estimated from the calculated $\mathrm{AUC}_{0-\infty}$ after administration of the different formulations.

\section{Statistical Analysis}

The estimated pharmacokinetic parameters for IBU after administration of the different formulations were calculated and were compared statistically using the independent samples Kruskal-Wallis test, utilizing SPSS, V25, to evaluate the pharmacokinetic behavior for the different formulations. $p$ of $<0.05$ was considered significant.

\section{Plasma Extraction and Analysis of Samples for IBU}

\section{Concentration}

The UFLC method described earlier for determination of IBU content was used for analysis of plasma samples after in vivo studies. However, the mobile phase composition of ACN:water:orthophosphoric acid (60:40:0.1 v/v) and a flow rate of $0.3 \mathrm{~mL} / \mathrm{min}$ were used for good resolution between the drug and internal standard peaks. Aliquots of $200 \mu \mathrm{L}$ of the rabbit plasma samples were spiked with the internal standard solution $(150 \mu \mathrm{L}$ of $10 \mu \mathrm{g} / \mathrm{mL}$ KET; Sigma, Switzerland) in methanol and then mixed (Vortex Genie; Scientific Industries, USA) for $30 \mathrm{~s}$ at 5,000 rpm. 


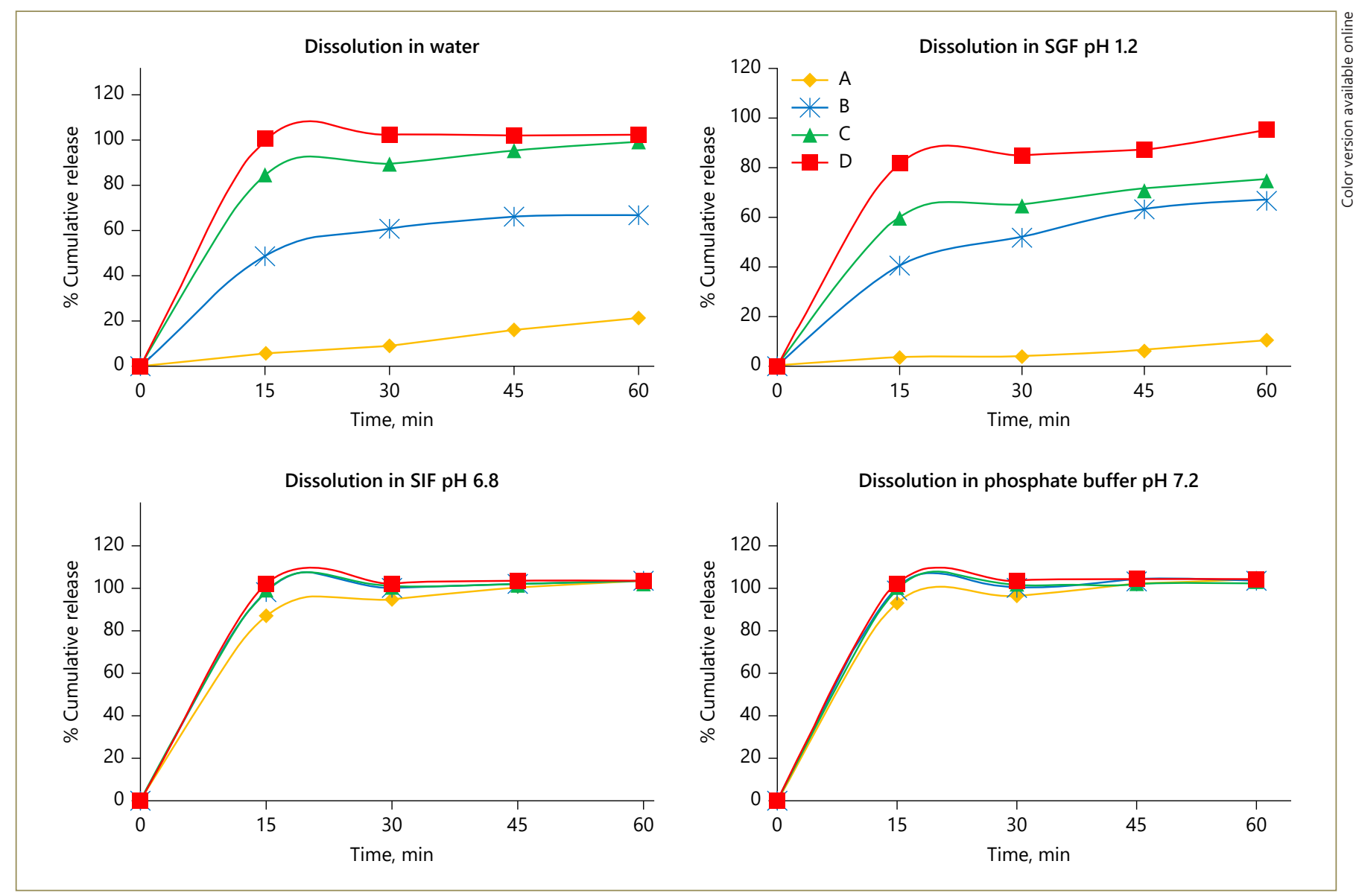

Fig. 1. Percent cumulative release profile of IBU in different dissolution media (IBU untreated (a); IBU marketed suspension (b); IBU:PVP K30 [1:2 w/w] ultrahomogenized nanoparticles (c); and IBU:PVP K30:TW [1:2:2 w/w] ultrahomogenized nanosuspension (d)). IBU, ibuprofen; TW, Tween 80; SGF, simulated gastric fluid; SIF, simulated intestinal fluid.

To each of the samples, $1 \mathrm{~mL}$ of ACN was added to precipitate plasma proteins. The samples were vortexed for $1 \mathrm{~min}$ and then centrifuged for $5 \mathrm{~min}$ at 10,000 rpm (Microfuge; Beckman Coulter, Germany). The supernatant was filtered through a $0.45-\mu$ syringe filter (Millipore), and $10 \mu \mathrm{L}$ of the sample was injected into the UFLC.

The calibration standards were prepared by spiking $200 \mu \mathrm{L}$ of blank rabbit plasma with known concentrations of IBU to obtain final concentrations of $0.25,0.5,1,5,10,20,30,40$, and 50.0 $\mu \mathrm{g} / \mathrm{mL}$. The internal standard solution was added to each of the calibration standard samples, and then the calibration standards were treated as the unknown samples. The calibration curves were constructed from the peak area ratios (IBU/KET) obtained for each of the calibration standards and the IBU concentration. IBU concentrations in the unknown samples obtained during the pharmacokinetic experiments were calculated from the equations of the calibration curves. The UFLC method developed in this work was validated according to the $\mathrm{ICH}$ guidelines [28].

\section{Results}

\section{Particle Size Analysis}

The particle size was significantly influenced by the homogenization pressure and number of homogenization cycles, as detailed previously [27]. IBU:PP (1:2 w/w) nanosuspensions exhibited mean particle size (513 \pm $25.62 \mathrm{~nm})$ and a narrow polydispersibility index (0.37). It was observed that adding TW to the drug:polymer mixture, IBU:PP:TW (1:2:2 w/w), further decreased the particle size $(132 \pm 11.47 \mathrm{~nm})$ with excellent redispersibility $(\mathrm{PI}=0.52)$, compared to untreated IBU which showed large particle size range 1,352 \pm 172.04 and high $\mathrm{PI}=1.89$.

\section{IBU Content Analysis}

The chromatographic method used for determination of IBU concentration in samples obtained from the in vi- 
Table 1. $\mathrm{DP}_{15}$ and $\mathrm{DP}_{60}$ parameters \pm standard deviation for untreated IBU, marketed IBU suspension, IBU freeze-dried nanoparticles, and IBU nanosuspension

\begin{tabular}{|c|c|c|}
\hline \multirow[t]{2}{*}{ Compound } & \multicolumn{2}{|c|}{ Dissolution parameters $^{\dagger \dagger \dagger}$} \\
\hline & $\mathrm{D}_{\mathrm{P} 1} 5$ & $\mathrm{DP}_{60}$ \\
\hline \multicolumn{3}{|l|}{ Dissolution medium $=$ water } \\
\hline Pure IBU & $6.23 \pm 4.21$ & $21.15 \pm 3.54$ \\
\hline IBU:PVP K30 (1:2 w/w) NP ${ }^{\dagger}$ & $85.71 \pm 1.89$ & $99.18 \pm 2.11$ \\
\hline IBU:PVP K30:T80 (1:2 w/w) NS ${ }^{\dagger \dagger}$ & $101.35 \pm 1.28$ & $102.61 \pm 1.07$ \\
\hline \multicolumn{3}{|l|}{ Dissolution medium $=\mathrm{SGF}$} \\
\hline Pure IBU & $3.57 \pm 3.68$ & $10.16 \pm 1.54$ \\
\hline IUB:PVP K30 (1:2 w/w) NP & $59.15 \pm 1.29$ & $75.14 \pm 1.28$ \\
\hline IBU:PVP K30:T80 (1:2 w/w) NS & $81.12 \pm 1.38$ & $95.62 \pm 1.57$ \\
\hline \multicolumn{3}{|l|}{ Dissolution medium $=\mathrm{SIF}$} \\
\hline Pure IBU & $87.78 \pm 3.61$ & $102.06 \pm 1.94$ \\
\hline IBU:PVP K30 (1:2 w/w) NP & $99.01 \pm 1.09$ & $102.89 \pm 1.18$ \\
\hline IBU:PVP K30:T80 (1:2 w/w) NS & $101.10 \pm 1.18$ & $103.2 \pm 1.17$ \\
\hline \multicolumn{3}{|c|}{ Dissolution medium = phosphate buffer $(\mathrm{pH} 7.2)$} \\
\hline Pure IBU & $93.78 \pm 3.61$ & $102.06 \pm 1.94$ \\
\hline IBU:PVP K30 (1:2 w/w) NP & $100.01 \pm 1.09$ & $103.89 \pm 1.18$ \\
\hline IBU:PVP K30:T80 (1:2 w/w) NS & $102.10 \pm 1.18$ & $103.2 \pm 1.17$ \\
\hline
\end{tabular}

IBU, ibuprofen; SGF, simulated gastric fluid; SIF, simulated intestinal fluid. $†$ NP indicates nanoparticles prepared by ultrahomogenization and freeze-drying. ${ }^{\dagger \dagger} \mathrm{NS}$ indicates nanosuspension prepared by ultrahomogenization. ${ }^{\dagger \dagger} n=3$.

tro experiments of content and dissolution analysis was linear in the range of $0.25-50 \mu \mathrm{g} / \mathrm{mL}\left(R^{2}=0.9994\right)$. The assay precision measured as the percent coefficient of variation $(\mathrm{CV} \%)$ determined over the concentration range of $10-30 \mu \mathrm{g} / \mathrm{mL}$ ranged from 0.33 to $1.3 \%$, for intraday precision $(n=3)$, and from 0.54 to $1.4 \%$, for the interday precision $(n=3)$. The assay accuracy measured as the mean \% relative deviation and determined in standard samples containing IBU in the range of 10-20 $\mu \mathrm{g} /$ $\mathrm{mL}$ was found to be between 1.17 and $2.34 \%(n=3)$. The contents of IBU in the nanosuspension, nanoparticles, and the marketed product were within $2 \%$ of the intended formulation composition and the labeled content for the marketed product.

\section{In vitro Dissolution}

Dissolution of the tested formulations was performed in various dissolution media (Fig. 1). The in vitro release profiles were evaluated in terms of the $\%$ of drug dissolved after 15- and 60-min intervals $\left(\mathrm{DP}_{15}\right.$ and $\left.\mathrm{DP}_{60}\right)$, and the data are presented in Table 1. The rate of dissolution for untreated IBU suspension was found to be too slow, reaching about $6.23 \%$ after $15 \mathrm{~min}$ and $21.15 \%$ after $1 \mathrm{~h}$. The dissolution rate of the marketed IBU suspension was retarded in simulated gastric fluid, with only about $17.47 \%$

In vivo Ibuprofen Nanosuspensions of the drug dissolved in $15 \mathrm{~min}$ and $35.77 \%$ dissolved at the end of $1 \mathrm{~h}$. Better dissolution was observed for the ultrahomogenized lyophilized nanoparticles $\left(\mathrm{DP}_{15}=\right.$ $60.18 \%)$ and ultrahomogenized nanosuspension $\left(\mathrm{DP}_{15}=\right.$ $80.16 \%$ ), as compared to the marketed suspension.

\section{Pharmacokinetic Experiments}

The chromatographic method developed for analysis of IBU extracted from plasma samples was found to be linear. The mean regression equation $(n=3)$ of IBU $(y=$ $0.9524 x-0.1646)$ was obtained by plotting the peak area ratios of IBU and KET (internal standard) versus concentration of IBU after extraction from plasma samples. The standard equation was found to be linear in the concentration range of $0.25-50 \mu \mathrm{g} / \mathrm{mL}\left(r^{2}=0.9994\right)$. The specificity of the method was verified by investigating the peak interference from the endogenous plasma substances. The blank plasma chromatogram was compared to that of plasma spiked and extracted samples of IBU. No endogenous interference was observed with both KET and IBU peaks. The retention times of KET and IBU were 3.27 and $6.25 \mathrm{~min}$, respectively. The drug peaks were well resolved (resolution $=4.53$ ) and symmetrical with a tailing factor of 1.18 and 1.23 for KET and IBU, respectively. The analyte samples were stable when stored for 3 days under 
Fig. 2. The mean IBU plasma concentration after oral administration of $25 \mathrm{mg} / \mathrm{kg}$ IBU in the form of nanosuspension, nanoparticles, unhomogenized mixture, marketed suspension, and untreated IBU suspension to rabbits. IBU, ibuprofen.

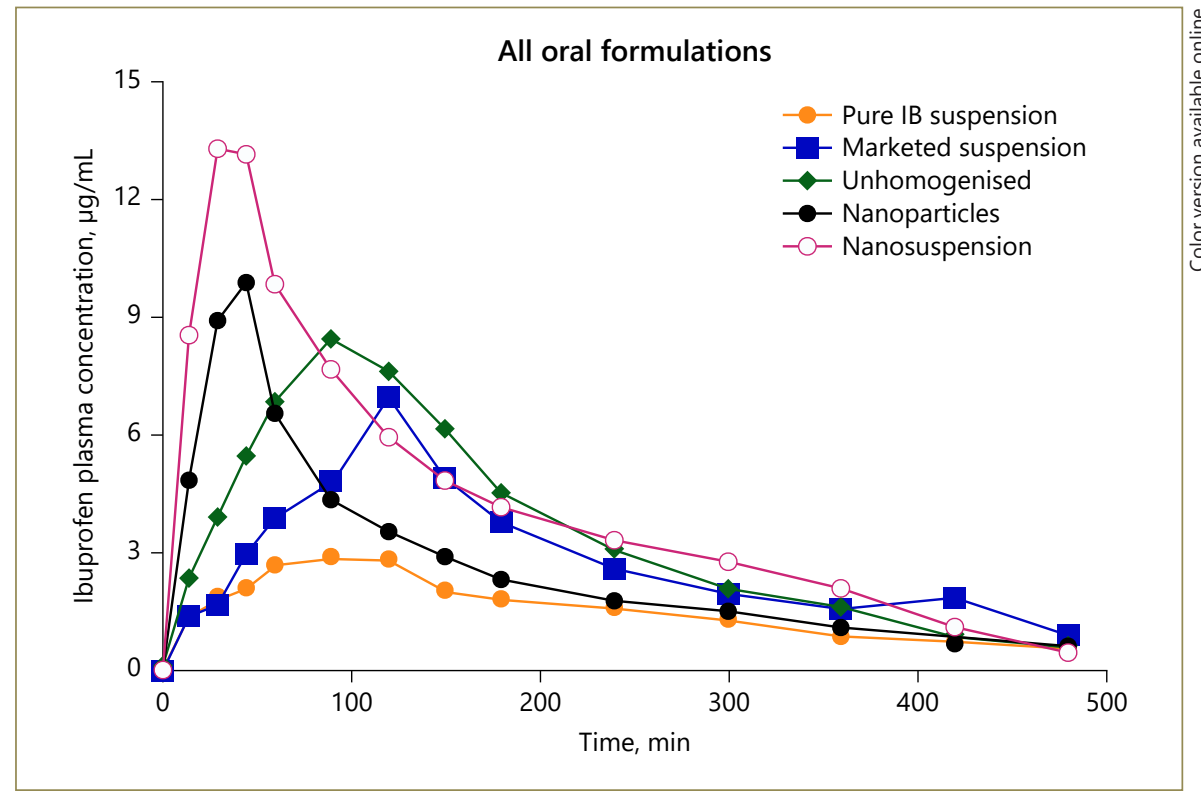

Table 2. IBU pharmacokinetic parameters estimated ( \pm standard deviation) after IV and oral administration of different formulations of IBU in rabbits

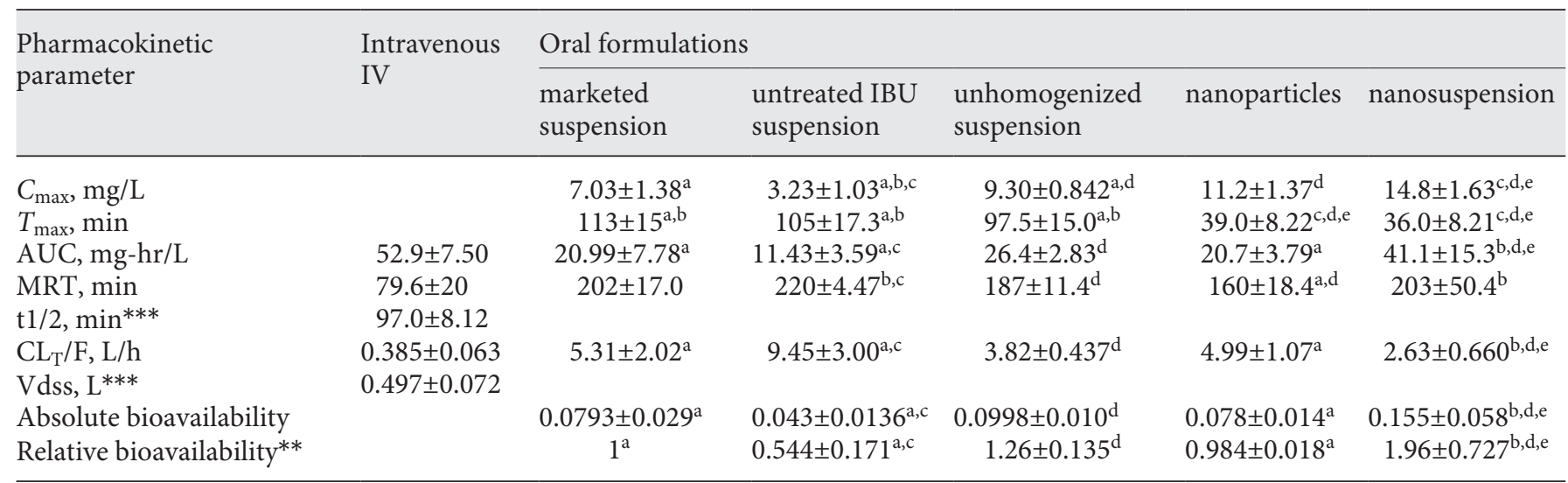

IBU, ibuprofen. * Statistical analysis was performed using the independent samples Kruskal-Wallis test by SPSS V $25 .{ }^{* *}$ Relative to the marketed IBU suspension. ${ }^{* * *}$ Calculated after IV administration. ${ }^{a}$ Significantly different from nanosuspension. ${ }^{\mathrm{b}}$ Significantly different from nanoparticles. ${ }^{c}$ Significantly different from unhomogenized. ${ }^{\mathrm{d}}$ Significantly different from untreated IBU suspension. e Significantly different from marketed IBU suspension.

refrigeration $\left(20 \pm 0.5^{\circ} \mathrm{C}\right)$ with a mean $\% \mathrm{RSD}<3$. The limit of quantification of IBU was $150 \mathrm{ng} / \mathrm{mL}(\mathrm{CV}<5 \%)$, and the minimum detectable level (LOD) was $75 \mathrm{ng} / \mathrm{mL}$. Recovery was obtained by comparing the peak area of known serum samples spiked with IBU to those of their respective aqueous solutions. The results showed an absolute recovery of $91.37 \pm 3.15 \%$.

After IV administration of hydroalcoholic solution of IBU, the plasma concentrations declined exponentially with a terminal half-life of $97 \mathrm{~min}$. On the other hand, the mean IBU plasma concentration-time profiles after administration of all oral formulations showed differences in the rate and extent of IBU absorption as presented in Figure 2. Table 2 includes a summary of the pharmacokinetic parameters calculated after administration of the IV and different oral IBU formulations. The maximum IBU plasma concentration $\left(C_{\max }\right)$ achieved after administration of the different formulations followed the following order: nanosuspension $>$ nanoparticle $>$ unhomogenized suspension $>$ marketed IBU suspension $>$ and untreated 
IBU suspension in water. The time to achieve the maximum concentration $\left(t_{\max }\right)$ was the shortest for nanosuspension followed by nanoparticle, then unhomogenized suspension, and then marketed IBU suspension and untreated IBU suspension in water. Taking the marketed IBU suspension as the reference formulation, the nanosuspension had the highest relative bioavailability $>$ unhomogenized suspension $>$ nanoparticle $>$ untreated IBU suspension in water.

\section{Discussion}

It has been reported that the transcellular pathway mainly absorbs particles through the cell membrane of enterocytes relying on their size and hydrophobicity [30]. Also, particles of smaller size are more easily absorbed by intestinal epithelial cells [31-33].

The optimal size reduction parameters and type of polymer and surfactant used greatly enhanced the properties of the resultant IBU nanoparticles. All tested formulations showed good dissolution rates in simulated intestinal fluid and buffer $\mathrm{pH}$ 7.2, with no significant differences between the dissolution pattern for the different formulations. This can be explained by the weak acidic nature of IBU ( $\mathrm{pKa}=4.43)$, which makes it ionized and more water soluble in neutral and alkaline medium. However, significant improvement in IBU dissolution at the acidic $\mathrm{pH}$ was observed for the ultrahomogenized formulations. This could be attributed to the reduction in particle size in submicron range, the amorphous nature of the material, and the increase of the hydrophilic environment around IBU particles due to the hydrophilic properties of the carrier polymer (PP). Both the developed IBU nanosuspension and the freeze-dried IBU nanoparticles exhibited excellent dissolution, making them suitable for liquid and solid dosage forms. The improved in vitro dissolution rate for IBU nanoparticles and nanosuspension was reflected in the in vivo behavior after oral administration in the rabbit.

The rate of IBU absorption after oral administration of the nanosuspension and the nanoparticles was faster than that of other oral formulations as indicated by the significantly shorter $t_{\max }$. This rapid oral absorption after administration of the nanosuspension resulted in significantly higher maximum IBU concentration compared to that after administration of the unhomogenized suspension, the marketed suspension, and the untreated IBU suspension in water. The calculated AUC after administration of the nanosuspension was significantly higher than the AUC calculated after administration of other formulations indicating significantly higher IBU bioavailability. The oral bioavailability of IBU increased by about 2-fold after administration of nanosuspension compared to that of marketed IBU oral suspension.

\section{Conclusion}

The results clearly indicate that the developed nanosuspension has a significantly faster rate of absorption compared to the other oral formulations tested. Accordingly, it can be concluded that formulation of poorly soluble drugs as nanosuspension using the ultrahomogenization technique is beneficial and leads to improvement in the efficacy of these drugs.

\section{Acknowledgements}

The authors are grateful to Research Sector, Kuwait University, for funding the project PP02/17. Special thanks to Mrs. Doha Nabeel and Mr. Saji Abraham for their technical support. They also thank the Animal Resource Center in the Health Science Center at Kuwait University for providing and caring for the animals utilized in this research project.

\section{Statement of Ethics}

The in vivo animal study protocol (PP 02/17) was reviewed and approved by the Animal Resources Centre (ARC), at Health Science center, Kuwait University and all procedures were conducted according to the Animal Research Reporting of In Vivo Experiments (ARRIVE) guidelines.

\section{Conflict of Interest Statement}

The authors do not have any of conflicts of interest related to the data submitted.

\section{Coauthors Statement}

The authors confirm that the contents and publication of the manuscript have been approved by all coauthors.

\begin{tabular}{|c|c|}
\hline References & $\begin{array}{l}1 \text { Raman S, Polli JE. Prediction of positive food } \\
\text { effect: bioavailability enhancement of BCS } \\
\text { class II drugs. Int J Pharm. 2016;506:110-5. } \\
2 \text { Kawabata Y, Wada K, Nakatani M, Yamada S, } \\
\text { Onoue S. Formulation design for poorly wa- } \\
\text { ter-soluble drugs based on biopharmaceutics } \\
\text { classification system: basic approaches and } \\
\text { practical applications. Int J Pharm. 2011;420: } \\
\text { 1-10. }\end{array}$ \\
\hline
\end{tabular}


3 Singh A, Worku ZA, Van den Mooter G. Oral formulation strategies to improve solubility of poorly water-soluble drugs. Expert Opin Drug Deliv. 2011;8:1361-78.

4 Stella VJ, Nti-Addae KW. Prodrug strategies to overcome poor water solubility. Adv Drug Deliv Rev. 2007;59:677-94.

5 Blagden N, de Matas M, Gavan PT, York P. Crystal engineering of active pharmaceutical ingredients to improve solubility and dissolution rates. Adv Drug Deliv Rev. 2007;59:61730.

6 Inam MA, Ouattara S, Frances C. Effects of concentration of dispersions on particle sizing during production of fine particles in wet grinding process. Powder Technol. 2011; 208(2):329-36.

7 Friedrich H, Nada A, Bodmeier R. Solid state and dissolution rate characterization of coground mixtures of nifedipine and hydrophilic carriers. Drug Dev Ind Pharm. 2005;31: 719-28.

8 Xia D, Cui F, Piao H, Cun D, Jiang Y, Ouyang $M$, et al. Effect of crystal size on the in vitro dissolution and oral absorption of nitrendipine in rats. Pharm Res. 2010;27:1965-76.

9 Badens E, Majerik V, Horváth G, Szokonya L, Bosc N, Teillaud E, et al. Comparison of solid dispersions produced by supercritical antisolvent and spray-freezing technologies. Int J Pharm. 2009;377:25-34.

10 Leuner C, Dressman J. Improving drug solubility for oral delivery using solid dispersions. Eur J Pharm Biopharm. 2000;50:47-60.

11 Hussain MD, Saxena V, Brausch JF, Talukder RM. Ibuprofen-phospholipid solid dispersions: improved dissolution and gastric tolerance. Int J Pharm. 2012;422:290-4.

12 Khattab IS, Nada A, Zaghloul AA. Physicochemical characterization of gliclazide-macrogol solid dispersion and tablets based on optimized dispersion. Drug Dev Ind Pharm. 2010;36:893-902.

13 Patel JS, Patel RP. Preparation, characterization and in vitro dissolution study of nitrazepam: cyclodextrin inclusion complex. J Pharm Bioallied Sci. 2012;4(Suppl 1):S106-7.
14 Vemavarapu C, Mollan MJ, Needham TE. Coprecipitation of pharmaceutical actives and their structurally related additives by the ress process. Powder Technol. 2009;189(3): 444-53.

15 Six K, Verreck G, Peeters J, Brewster M, Van Den Mooter G. Increased physical stability and improved dissolution properties of itraconazole, a class ii drug, by solid dispersions that combine fast- and slow-dissolving polymers. J Pharm Sci. 2004;93:124-31.

16 Lee J, Lee SJ, Choi JY, Yoo JY, Ahn CH. Amphiphilic amino acid copolymers as stabilizers for the preparation of nanocrystal dispersion. Eur J Pharm Sci. 2005;24:441-9.

17 Lee J, Choi JY, Park CH. Characteristics of polymers enabling nano-comminution of water-insoluble drugs. Int J Pharm. 2008;355: 328-36.

18 Ghosh I, Bose S, Vippagunta R, Harmon F. Nanosuspension for improving the bioavailability of a poorly soluble drug and screening of stabilizing agents to inhibit crystal growth Int J Pharm. 2011;409:260-8.

19 Koh PT, Chuah JN, Talekar M, Gorajana A, Garg S. Formulation development and dissolution rate enhancement of efavirenz by solid dispersion systems. Indian J Pharm Sci. 2013; 75:291-301

20 Yadav GV, Singh SR. Nanosuspension. A promising drug delivery system. Pharmacophore. 2012;3:217-43.

21 Lai F, Sinico C, Ennas G, Marongiu F, Marongiu G. Fadda AM: diclofenac nanosuspensions: Influence of preparation procedure and crystal form on drug dissolution behaviour. Int J Pharm. 2009;373:124-32.

22 Wang Y, Li X, Wang L, Xu Y, Cheng X, Wei P. Formulation and pharmacokinetic evaluation of a paclitaxel nanosuspension for intravenous delivery. Int J Nanomedicine. 2011;6: 1497-507.

23 Jacobs C, Müller RH. Production and characterization of a budesonide nanosuspension for pulmonary administration. Pharm Res. 2002;19:189-94.
24 Peters K, Leitzke S, Diederichs JE, Borner K, Hahn H, Müller RH, et al. Preparation of a clofazimine nanosuspension for intravenous use and evaluation of its therapeutic efficacy in murine mycobacterium avium infection. J Antimicrob Chemother. 2000;45:77-83.

25 Wang Y, Pi C, Feng X, Hou Y, Zhao L, Wei Y. The influence of nanoparticle properties on oral bioavailability of drugs. Int J Nanomedicine. 2020;15:6295-310.

26 Montero N, Alhajj MJ, Sierra M, Oñate-Garzon J, Yarce CJ, Salamanca CH. Development of polyelectrolyte complex nanoparticlesPECNS loaded with ampicillin by means of polyelectrolyte complexation and ultra-high pressure homogenization (UHPH). Polymers. 2020;12:1168.

27 Nada A, Bandarkar F, Al-Basarah Y. Formulation of ibuprofen nanoparticles and nanosuspensions with enhanced dissolution rate using ultra-homogenization technique. Asian J Pharmaceutics. 2017;11(Suppl 1):S14-S23.

28 FDA-bioanalytical method validation guidance for industry. 2001. Available from: https://www.fda.gov/files/drugs/published/ Bioanalytical-Method-Validation-Guidancefor-Industry.pdf.

29 Hedaya MA. Basic pharmacokinetics. Boca Raton: CRC Press. 2012

30 Luo YY, Xiong XY, Tian Y, Li ZL, Gong YC, Li YP. A review of biodegradable polymeric systems for oral insulin delivery. Drug Deliv. 2016;23:1882-91.

$31 \mathrm{Hu}$ L, Tang X, Cui F. Solid lipid nanoparticles (SLNs) to improve oral bioavailability of poorly soluble drugs. J Pharm Pharmacol. 2004;56:1527-35.

32 Li H, Chen M, Su Z, Sun M, Ping Q. Size-exclusive effect of nanostructured lipid carriers on oral drug delivery. Int J Pharm. 2016;511: 524-37.

33 Zhao Z, Hu Y, Hoerle R, Devine M, Raleigh $\mathrm{M}$, Pentel P, et al. A nanoparticle-based nicotine vaccine and the influence of particle size on its immunogenicity and efficacy. Nanomedicine. 2017;13:443-54. 